\title{
New Perspective in Regional and Residual Separation of Gravity and Magnetic Data Processing
}

\author{
Wiji Raharjo', Indriati Retno Palupi², Oktavia Dewi Alfiani' ${ }^{3}$ \\ 1,2 Geophysics Engineering, Universitas Pembangunan Nasional “Veteran” Yogyakarta, Indonesia. \\ ${ }^{3}$ Geomatics Engineering, Universitas Pembangunan Nasional "Veteran" Yogyakarta, Indonesia.
}

\begin{abstract}
Separation between Regional and Residual anomaly in Gravity and Magnetic data processing is very important to get the best result in geological interpretation. Several methods were used to solve this problem like upward continuation and polynomial fitting. With the same principle, 2D FFT is applied by make an interactive tool based on Matlab Language Programming, named "Oasis Ala-Ala". It adopts the algorithm from software Oasis. It started with make visualization map or the original data, then the map divide into some grids. Each of grid contain gravity or magnetic data. Then it transformed from special to wavenumber domain. After that, it convolves with our own filter matrix. And the last step is inverse it to get the regional and residual anomaly map. However, Matlab is powerful in facilitate this process in the GUI Toolbox. One important thing is the size of gravity and magnetic data. It will improve to Filter matrix size before do inverse process.
\end{abstract}

Keywords: Gravity, Magnetic, Regional and Residual Separation, 2D FFT

This is an open access article under the CC-BY-NC license

\section{INTRODUCTION}

Gravity and magnetic method are part of geophysical way to get the description of the subsurface based on gravity acceleration and magnetization parameter in the medium, especially metal for the magnetic. Furthermore, the description of the gravity and magnetic is interpreted from the density and susceptibility pattern as the result of the data processing. The density shows the mass distribution in one volume unit, while the susceptibility shows the magnetization ability of the metal. Both of them are often used as a preliminary survey before the exploration, especially in mining, oil and gas.

The processing data of gravity and magnetic method are similar. After the gravity acceleration and magnetization are collected ( $\mathrm{g}$ abl and $\mathrm{H}$ ), they are filtered with some filters to get the separation between the regional and residual anomaly. It is an important thing in anomaly's depth calculation. The gravity and magnetic are influenced by the spatial factor, so the filtering should be done in 2 Dimension or in a Map data. The anomaly's depth calculation is processed after the data converted from spatial to wavenumber domain. Some filters can be applied to the data are upward continuation, polynomial fitting and 2D Fast Fourier Transform (2D FFT). They have their own mathematical equation, but it is still in the same concept to separate regional and residual anomaly of the data. Because the data is based on the map, the filter should be in 2 Dimensional also. Several software use Upward Continuation as the method, like in Oasis Montaj. Different with the Oasis Montaj, this research will gives the description about how the mathematical process of the 2D FFT filter to separate the regional and residual anomaly. Furthermore, this concept is easy and can be done in Matlab and also can be applied in the academic curriculum to increase the knowledge especially for the student. 


\section{METHODOLOGY}

The gravity coming from the Newton Law. In the Newton Law, the force between two mass (the earth's core and everything located in the surface) depend on their distance and the density. The Newton Law then can be seen in equation (1)

$$
F=G \frac{\underline{m}_{1} \underline{m_{2}}}{r^{2}}
$$

where $\mathrm{F}$ is the force $(\mathrm{N}), \mathrm{G}$ is the gravitation constant, $\mathrm{m} 1$ and $\mathrm{m} 2$ are the mass of the earth and the anomaly and the $r$ is the distance between $\mathrm{m} 1$ and $\mathrm{m} 2$.

Then the earth mass is assumed constant, so the equation (1) can be rewritten as :

$$
F=G \frac{M m_{2}}{r^{2}}=g \frac{m_{2}}{r^{2}}=m_{2} a
$$

where a is the gravitational acceleration. As we know that the gravitational acceleration has the universal value, that is $9.8 \mathrm{~m} / \mathrm{s}^{2}$. Otherwise, the fact, the gravitational value is different in each place in the earth. It depends on the longitude and latitude location and also the density of the medium. Therefore, the equation (2) run into the detail equation, like in equation(3)

$$
g=G \frac{\rho V}{r^{2}}
$$

where $\mathrm{g}$ is the gravitational acceleration in the certain area, is the density and $\mathrm{V}$ is earth's volume. Then $\mathrm{V}$ is assumed as a constant value (Valenta, 2015)

The gravitational acceleration respond can be seen in the Figure 1

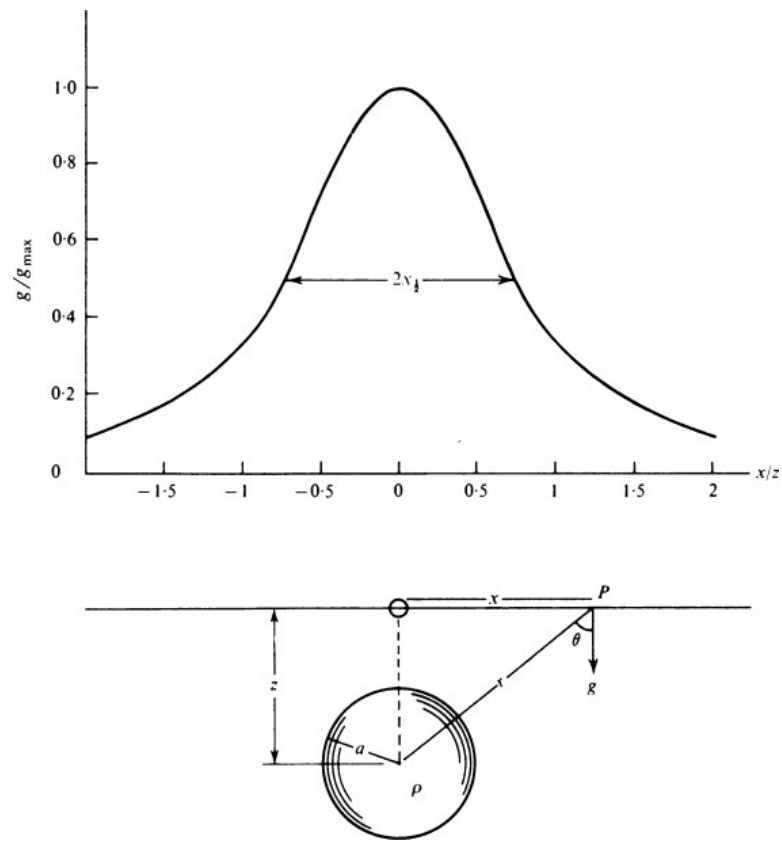

Figure 1. The gravitational acceleration (Upper) based on the anomaly location (Lower) (Telford et al 1990 in Valenta, 2015) 

Based on Figure 1, we can see that the density, its location and size of the area or even the volume are very influenced to the gravitational acceleration respond. It increases appropriate to the centre location of the anomaly. To get the geological model, the density should be modelled well by the inversion method, and it is the goal of the gravity processing data. After the depth of the anomaly and the density are described well, the geology interpretation should be easier as mentioned by Benson and Floyd (2000). They are success in identifying graben pattern in the Mosida Hills, Utah, based on the density model as the result of gravity processing data like in Figure 2

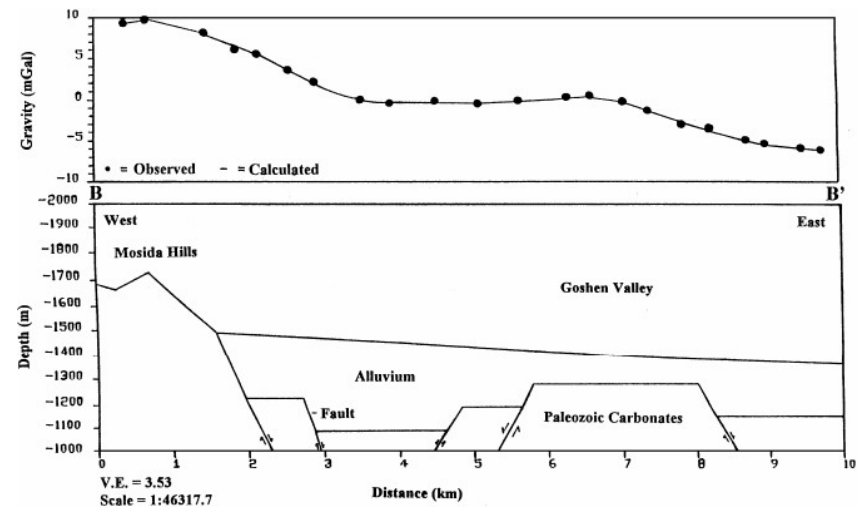

Figure 2. Residual anomaly respond (upper) and the graben structure (lower) (Benson and Floyd, 2000)

The second method is the magnetic method. Its values depends on the differences of the magnetic intensity in the subsurface, so it can be used to identify the mineral that usually include into the metal. The goal of this method is the susceptibility value. It represents the ability of the metal to be magnetized. The magnetic equation can be seen in equation (4)

$$
M=k H
$$

where $\mathrm{M}$ is the magnetic intensity, $\mathrm{k}$ is the susceptibility and $\mathrm{H}$ is the magnetic field.

Based on the equation (4), it is clear that the magnetic intensity is the independence variable, it is influenced by the susceptibility and the magnetic field. Furthermore it are influenced by the declination and inclination angel too. Declination angel is measured from the North direction of the earth to the resultant vector of the magnetic field in the surface, while the inclination is measured from the resultant vector of the magnetic field in the surface to the magnetic field in the certain depth (Valenta, 2015).

There are three types of magnetization, they are diamagnetic, paramagnetic and ferromagnetic. They depend on the mineral type in the subsurface. The diamagnetic is identic with the susceptibility value less than zero and there is no remanent magnetic or the magnetization mass will reduce due to the decrease of atomic interaction in the mineral. It does not depend to the temperature too. The paramagnetic is identic with the susceptibility value more than zero, there is no interaction between the atom in the mineral and the magnetic filed's direction are similar. While, the ferromagnetic has the same character with the paramagnetic but there is interaction between the atom in the mineral (Erkan, 2008).

As mention before, the density and susceptibility become the important parameter in gravity and magnetic method. Their modelling helped the user having good interpretation in geology. It is gotten from the inversion processing data. Unfortunately, some challenges often coming in the inversion process. One of them is the determination about the depth information in the modelling. This problem can be solved with the power spectrum way that is calculated in the map in the 
wavenumber domain. Based on the equation (3) and (4), density and the magnetic intensity are the data, while density and the susceptibility be the parameter model. The others parameter then be the Forward Modelling parameter and they are called the Kernell Matrix. All of the information supporting to get the accurate model as the result (Butler and Sinha, 2011).

Generally, the basic concept of the inverse modelling is like in equation (5)

$$
d=G m
$$

where $d$ is the data, $G$ is kernel matrix and $m$ is the parameter model. The Kernell matrix then developing to the singular matrix and the mathematical solution can be seen in equation (6)

$$
m=G^{T}\left(G G^{T}+\lambda I\right)^{-1} d
$$

However, the equation (6) uses the least square method, with as the perturbation factor and I is the matrix identity (Grandis and Dahrin, 2014).

Inverse method in this paper uses as the validation way of the 2D FFT filter. For the 2D FFT filter itself is still similar with the 1D FFT that is usually used in seismic method to convert the time domain into frequency domain. 2D FFT read the 2 Dimensional data, divide them into several grid and then transform the value in each grid sequentially. In Figure 3, we can see the illustration of 2 Dimensional data

\begin{tabular}{|c|c|c|c|}
\hline B1 & B2 & B3 & B4 \\
\hline B5 & B6 & B7 & B8 \\
\hline B9 & B10 & B11 & B12 \\
\hline B13 & B14 & B15 & B16 \\
\hline B17 & B18 & B19 & B20 \\
\hline B21 & B22 & B23 & B24 \\
\hline
\end{tabular}

Figure 3. illustration of 2 Dimensional data in certain grid

In 2D FFT, the value in each block read as pixel and it will be converted to the magnitude value based on the equation (7)

$$
f(u, v)=\iint f(x, y) e^{-i 2 \pi\left(u x+v_{y}\right)} d x d y
$$

where $u$ and $v$ are assumed as the angular frequency of $x$ and $y$.

Rauh and Arce applied scaling and shearing in 2D FFT processing in the window's length to get the good result of the image like in Figure (4).

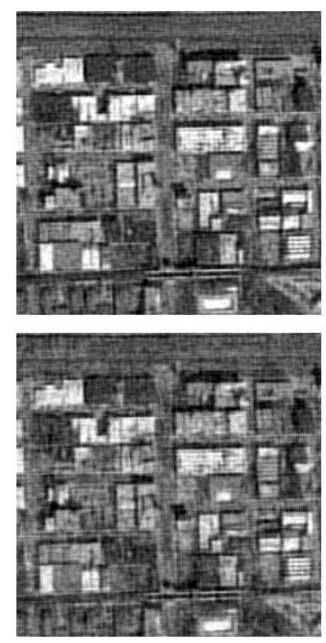

Figure 4. Image before 2D FFT (upper) and after 2D FFT (lower) 
Based on Figure 4, the image after 2D FFT is more contrast than before. The window's length specifying the duration time of the processing and also the its result. For the information, the little size of window's length is not always resulting the good model, so the trial and error is needed to get the best one.

\section{RESULT AND DISCUSSION}

This research started by collecting the gravity data form TOPEX website. We choose Yogyakarta area as the data. As we know, the data from Topex is included to the free air anomaly gravitational data. It should be convert to the Bouger anomaly first, but in this paper we just want to show how the 2D FFT works well to the data, so the data is assumed as Bouger anomaly data.

Overall, we make the simple GUI Toolbox based on the Matlab language programming. We choose Matlab because it has complete mathematical tools. The descrption of the GUI Toolbox can be seen in Figure 5

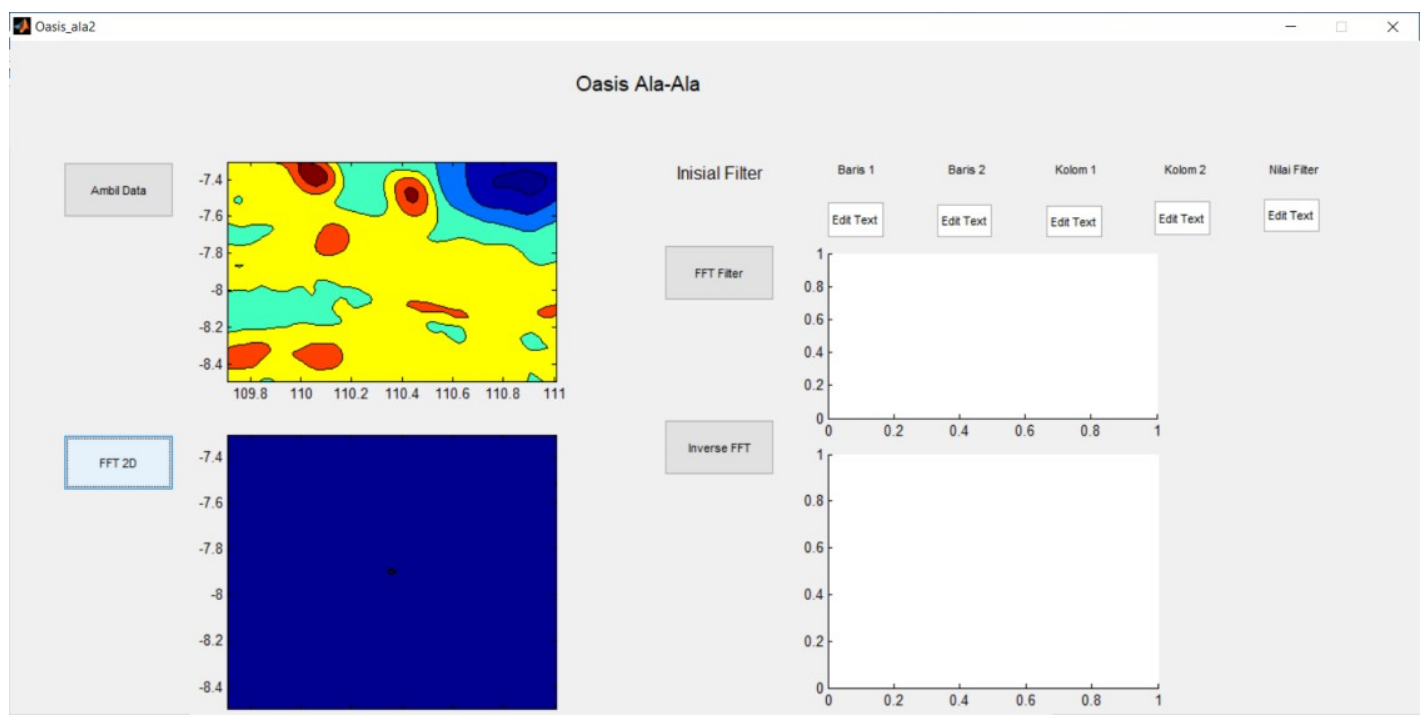

Figure 5. GUI Toolbox

We design the GUI Toolbox with several fitures like in the Figure 5. For the First, the data can be loaded by the "Ambil Data" button. The data should be saved in the same folder with the matlab document. We can see in the Figure 5, the Yogyakarta map is tend to the homogenous medium. It is represent by the homogenous colour of the map. After that, 2D FFT is applied to the map by click the "FFT 2D" button. The map now is in the wavenumber domain and power spectrum can be calculated by make the slice first on the map.

The wavenumber has no unit, so to make the map in the frequency domain, the map then convolve with the filter matrix. The filter matrix should have the same size with the map. The design of the filter matrix depend on the anomaly will be enhanced. It can be solved by input the initial filter to develop the matrix filter by click the "FFT Filter" button. The result can be seen in the Figure 6. 


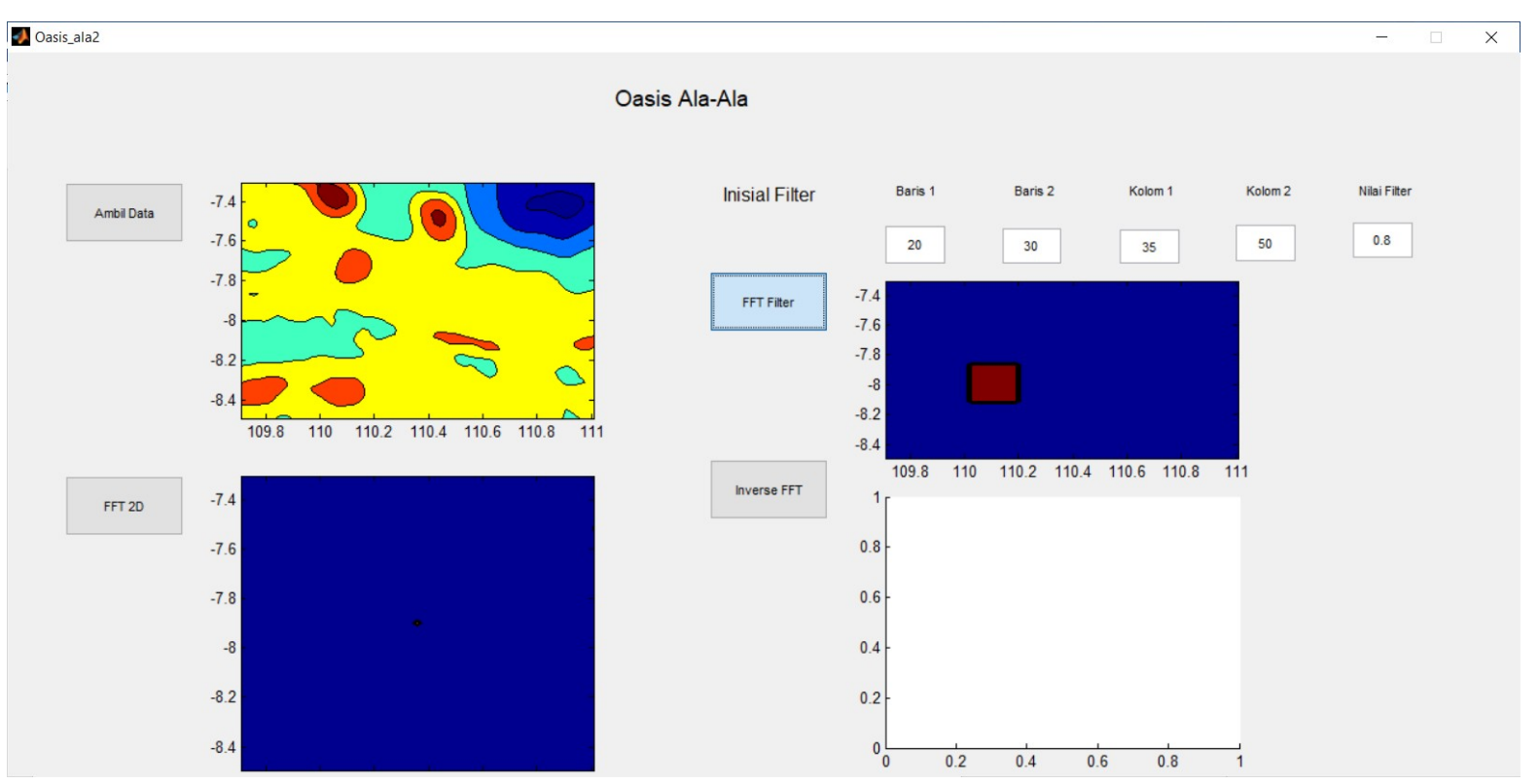

Figure 6. The matrix filter design

The matrix filter design will determine the result of regional and residual separation. Like in Figure 6, we place the high filter that is represented by the red colour (0.8), and the blue colour for the low value (0.3). After that, the convolution between the map and the matrix filter inverse by click the "Inverse FFT" button to get the regional and residual anomaly separation and the result can be seen in Figure 7. The filter value ranging from 0 to 1.

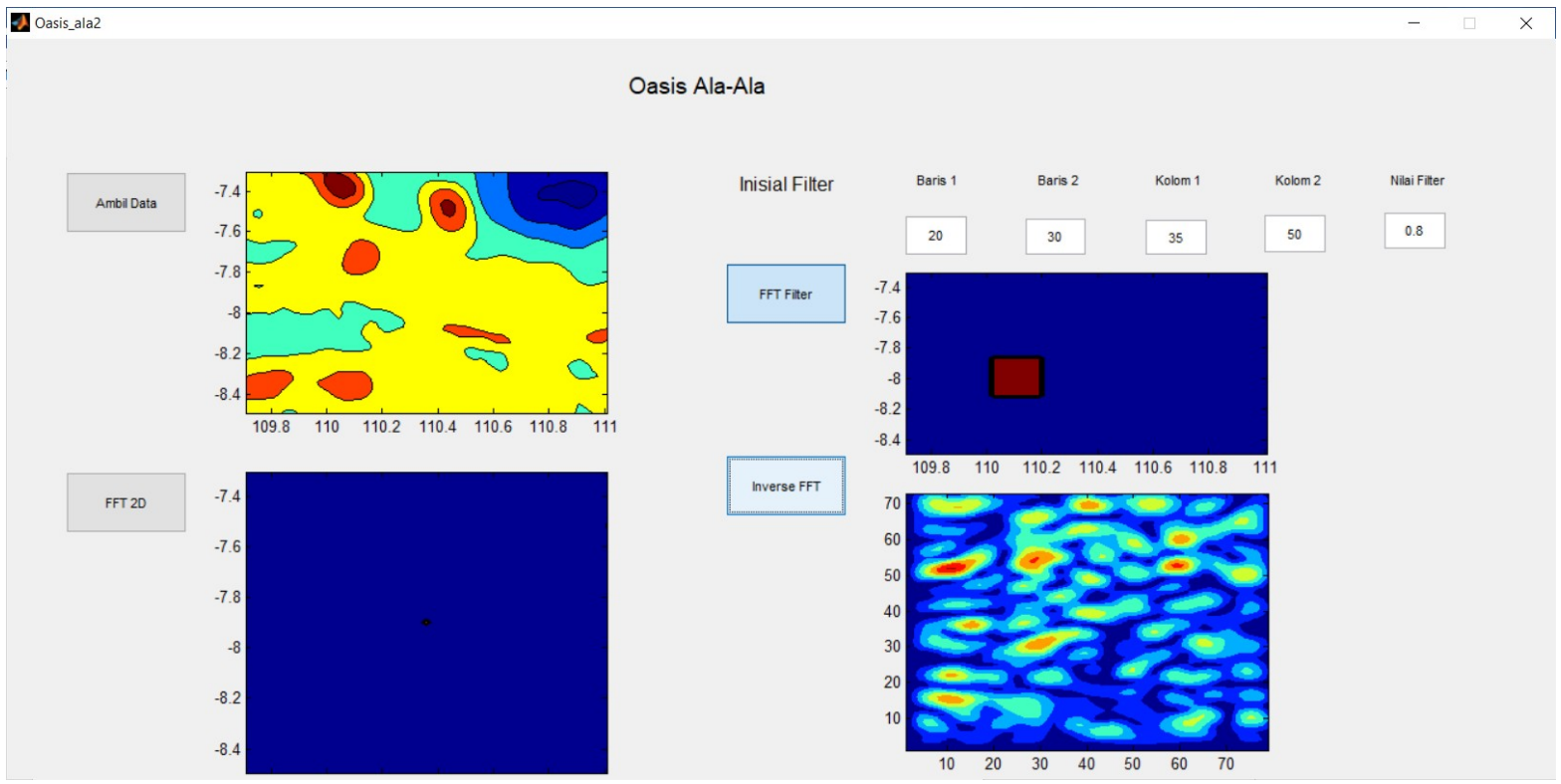

Figure 7. The regional and residual anomaly result

For the geoscience, there is no information based on the regional and the residual anomaly result in Figure 7. It means that we should replacement the high filter in the other location. This process can be occurred more than once or called trial and error process. After several tests, finally we have a good result like in Figure 8 


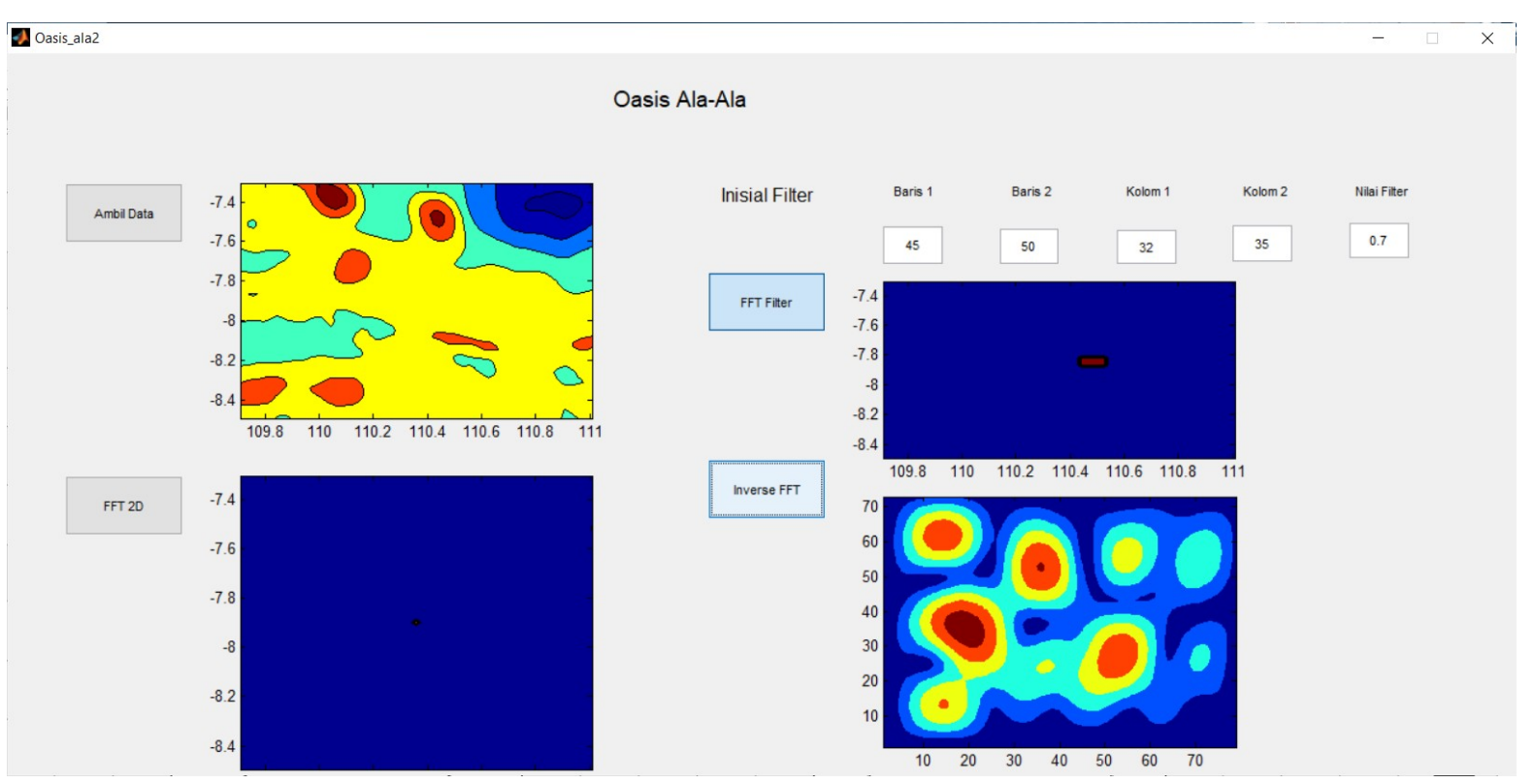

Figure 8. The Regional and residual anomaly result

\section{CONCLUSION}

2D FFT is alternative way to separate regional and residual anomaly map of gravity and magnetic data. It can be made easily using the matlab by the GUI Toolbox. 2D FFT divide the map into several grid and read them as the pixel value than with the mathematical equation like in 1D FFT the map converting from the spatial into the wavenumber domain. Power spectrum then calculated by make the slice in the wavenumber map first. It is important in the inversion processing to give the depth information. Wavenumber has no unit, so it convolves with the filter matrix that has the same size with the map. After that, inverse method is applied to get the regional and residual separation. However, trial and error way is needed to get the best result.

\section{REFERENCE}

Benson, Alvin K dan Andrew R Floyd. 2000. Application of Gravity and Magnetic Methods to Assess Geological Hazard and Natural Resource Potential in The Mosida Hills, Utah Country, Utah. Geophysics Vol 65 No 5

Butler, S.L dan G Sinha. 2011. Forward Modelling of Applied Geophysics Methods Using Comsol and Comparison with Analytical and Laboratory Analog Models. Department of Geological Science, University of Saskatchewon, Canada

Erkan, Kamil. 2008. A Comparative Overview of Geophysical Methods. Geodetic Science and Surveying, The Ohio State University.

Foulger, G.R dan C. Peirce. Geophysical Methods in Geology.

Grandis, Hendra dan Darharta Dahrin. 2014. Constrained Two-Dimensional Inversion of Gravity Data. Journal Math Fundamental Science Vol 46 No 1

Rauh, Andre dan Gonzalo R Arce. Sparse 2D Fast Fourier Transform. Proceeding of The $10^{\text {th }}$ International Conference on Sampling Theory and Application

Valenta, Jan. 2015. Introduction to Geophysics-Lecture Notes. Czech Republic Development Cooperation 